\title{
Optical Communication with WDM based FSO over Varying Weather Conditions
}

\author{
Rinkesh Mittal, Agrima Kukkar, P. N. Hrisheekesha
}

\begin{abstract}
These days, Free Space Optics (FSO) has become a prominent mechanism due to its economic nature, broad-bandwidth access technique. The effects of unpredictable weather are difficult to deal. In order to draft the most effective system, Single beam and multiple beam FSO systems are developed which can tackle the atmospheric weather condition's effects on signal. In this dissertation, a practical review on communication channels (FSO) is presented. These channels are used in WDM. It was obtained that the traditional Wavelength Division Multiplexing (WDM) based FSO system utilized the NRZ encoding method which was less efficient and it was suitable for communication channel which supports 16 transmitters and receivers only. This work is organized to achieve an objective to perform some enhancements in traditional WDM system by replacing NRZ encoding scheme with MDRZ encoding and also the channel size is increased to the 32.
\end{abstract}

Keywords-Free Space Optic, WDM, NRZ, Duobinary Return to Zero, Modified Duobinary Return to Zeroformat.

\section{INTRODUCTION}

For all the techniques used in the optical access networks, FSO is proved to be the optimistic technology [1]. It is a way of sending visible or IR rays into the atmosphere after modulating the signal in a line of sight communication method. Therefore, there is no need of any cables such as fiber optics. The FSO system is possible to develop at 1550 $\mathrm{nm}$ because of its small attenuation window and access to components using high-speed laser semi-controller technology [2]. For this system, due to the advantage ofhigh data rate and flexibility, optical fiber communication is able to solve the issues occurred. The FSO system consists of infrared rays as well as visible rays whichis transmitted during the communication process. But this system consists of disadvantage of poor connectivity and performance [3]. This combination of laser beam and other rays may deduct the turbulent effects in atmosphere. For example, power loss in detector [4]. This system also produces geometrical losses despite of atmospheric losses [3]. The FSO system analyzed with multiples beams excludes intermittent physical obstacles as birds and insects, sparkling water, rain, snow and "Fail Safe" [5]. For example, due to the low visibility during fog in coastal areas, FSO system can be used to ensure greater availability of connections [6].

The interconnections with one-beam transceiver in the heavy rain are less efficient for a FSO communication system. To

Revised Manuscript Received on June 15, 2019

Dr. Rinkesh Mittal, Electronics and communication Engineering,

Chandigarh Engineering college,Landran, Mohali, Punjab

Agrima Kukkar, Electronics andCommunication Engineering ,

Chandigarh EngineeringCollege, Landran, Mohali, Punjab.

Dr. P. N. Hrisheekesha,Electronics and communication Engineering

Chandigarh Engineering college Landran, Mohali, Punjab increase the link performance, it could be possible to use several beams in the transmitter and receiver [7]. The optical signal is smooth by weather such as fog, low winds, and rain and snow dust [7]. Optical signal attenuation depends on the size and velocity of raindrops falling [8-10]. Compare to light rain, heavy rain produces higher attenuations [11-12]. In bad weather conditions, a priority optimization is helpful to improve the FSO connection performance [13].

Free Space optics technology that is based on wave division multiplexing could be enhanced up to its maximum range of linking data. Therefore, the quality of signal is controlled by the design of wave division multiplexer as well as the channel used in FSO during transmission.

\section{II- ATMOSPHERIC EFFECT ON FSO SYSTEM}

Attenuation of signal due to rain is the concept also known as "Non Selective Dispersion". Due to atmosphere conditions like dust, rain, snow, fog, system performance may degrade. The main reason of signal distortion are geometry loss and atmospheric attenuation but there are few more facts like scintillation and turbulence too [14]. Temperate regions are affected by heavy snow and the tropical region by heavy rain. When from the optical beam there is scattering of large rain drops then the effect is known as scattering. Factors affect the performance of the connection and result in power and $\mathrm{Q}$ factor degradation. The power received is asfollows:

$$
P_{\text {Received }}=P_{\text {Transmitted }} \quad \frac{d_{r}^{2}}{\left(d_{T}+\theta R\right)^{2}} 10^{-\alpha_{10}^{R}}
$$

(1) Where, $\mathrm{dr}$-receiver aperture diameter incm,

(2) dT- transmitter aperture diameter incm

(3) $\theta \mathrm{R}$ - beam divergence angle in $\mathrm{mrad}$

(4) R- linkrange

(5) $\alpha$-atmospheric attenuationcoefficient

This paper [15] addresses the questions raised during designing a high-spectrally proficient 40Giga byte per second system with the analysis of the DRZ called scheme of carrier compression in optical communication. While Modified Duobinary Return to Zero and duobinary return to zero effectively suppress the discrete frequency tones of the RZ signal spectrum, the later has the advantage of smaller time jitters and amplitude distortions. In addition, [16] both 


\section{Optical Communication With Wdm Based Fso Over Varying Weather Conditions}

Modified Duobinary Return to Zero and duobinary return to zero are operating smoothly, even moderately greater standard channel power compared to RZ. Substantial data in the format of a limited number of channels was examined in DWDM technique or for a typical data rate of less than 40 Gbps (Sikdar et al., 2012; Tan \& Pincemin, 2009). A 40 Gbps modulated system using MDRZ technique has been tried to evaluate from previous techniques [17]. The study examines further the potential of integrating unequal canal spacing to remove FWM in optical links. Various techniques used for WDM are asfollows:

- $\quad$ Non-back to zero formats - NRZ format was extensively employed in currently deployed systems due to its simple configuration, as depicted in $1^{\text {st }}$ figure. $\mathrm{CW}$ laser provides signals of on - off mechanism of $40-\mathrm{GHz}$ Non Return to Zero data stream-driven for Mach - Zehnder Intensity Modulator

- $\quad$ RZ double-binary format - It owes its superiority over Non-Return to Zero as it has a smaller spectral bandwidth and improves residual CD tolerance. This alleviates the requirements for the integrated DCM module on the recipient side. In addition, its portable spectrum makes it compatible with $50 \mathrm{GHz}$ ITU grid and conforms the 40 Gbps system with the already existing 10 Gbps longitudinal WDM system (Xu, Wang, Chowdhury, Yu, \& Chang, 2006; Yonenaga \& Kuwano, 1997) and permits an easier up-grade to 40 Gbps network by just interchanging the transmitters without making significant changes in the receiver and transmission linedesign.

- MDRZ has a much smaller visual bandwidth across the DRZ, which means more tolerance for dispersion and higher non-linear fibre (Miyamoto et al., 2001; Xu et al., 2006). The stages of two groups of "one" in this format are flipped into an isolated "zero," leading to a regeneration of the ghost pulse due to four-wave intrachannel mixing. In order to obtain this signal, however, it needs two optical modulators: one to generate the dual binary signal from the NRZ and the other for carvingNRZ to the RZ signal, thus increasing the transmitter costs. Figure shows the design of the duobinary transmitter modified by 40 Gbps.

A delay and subtract circuit is required to generate Duobinary Non Return to Zero from the Duobinary Return to Zero technique. This block's output signals the first MZM with its output linked to a second modulator that is driven by a $40 \mathrm{GHz}$ frequency and $-90^{\circ}$ sinusoidal electric signal. We only change the phase for bits 1 with the bit " 0 " in duobinary signal case. For MDRZ, bit " 1 " is exchanged with 0 to $\mathrm{T}$ bits. As the signals which have zero phases in a constant manner, we introduced a $180^{\circ}$ phase shift between the " 1 ". This further alters the carrier of the duobinary signal to bedeleted.

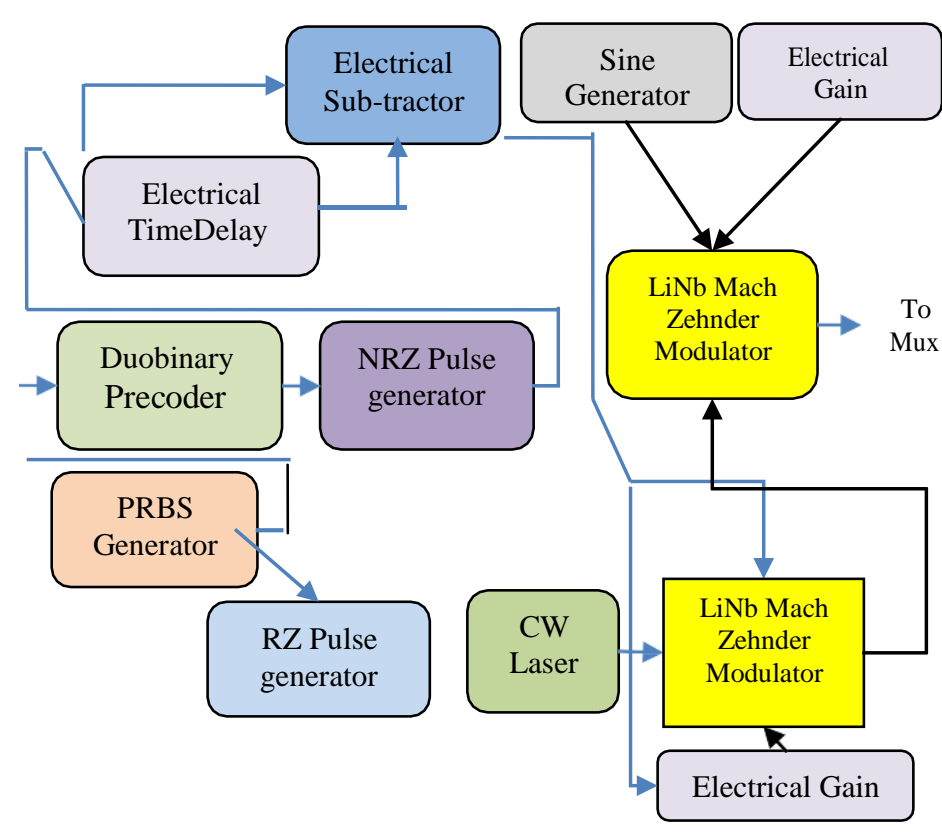

Figure 1: Block diagram of modified duo-binary transmitter (MDRZ)

\section{PROBLEMFORMULATION}

A significant problem in the optical communication system is strong atmospheric instability. The transmitter is a 20Gbps PRBS, which uses NRZ format to modulate the data. But NRZ format used in these systems for modulation do not generate results accordingly. Moreover, the traditional system was designed for 16 channels with one optical splitter and one optical combiner. Base station (BS) consists of 16 transmitters which generate optical transmitters with a variety of wavelengths from 1538 to $1550 \mathrm{~nm}$ with $100 \mathrm{GHz}$ channel distance. Thus an improved version of communication channel in the WDM system must be required todevelop.

\section{PROPOSED WORK}

After studying the previous work in the field of optical communications, it was found to be possible to analyze the various communications channels and to implement NRZ coding in traditional research work. NRZ is a modulation technique which is an enhancement of RZ modulation coding scheme. With the development of optical filter, there is no add up in modulation technique such as Non Return to Zero. A number of techniques are proposed, but no technique can perform accordingly to deliver a high data rate at a high speed.

The above figure 2 illustrates the proposed optical model in which we worked with. In the proposed modulation work schemes such as the MDRZ, traditional modulation techniques are replaced with the NRZ etc. 


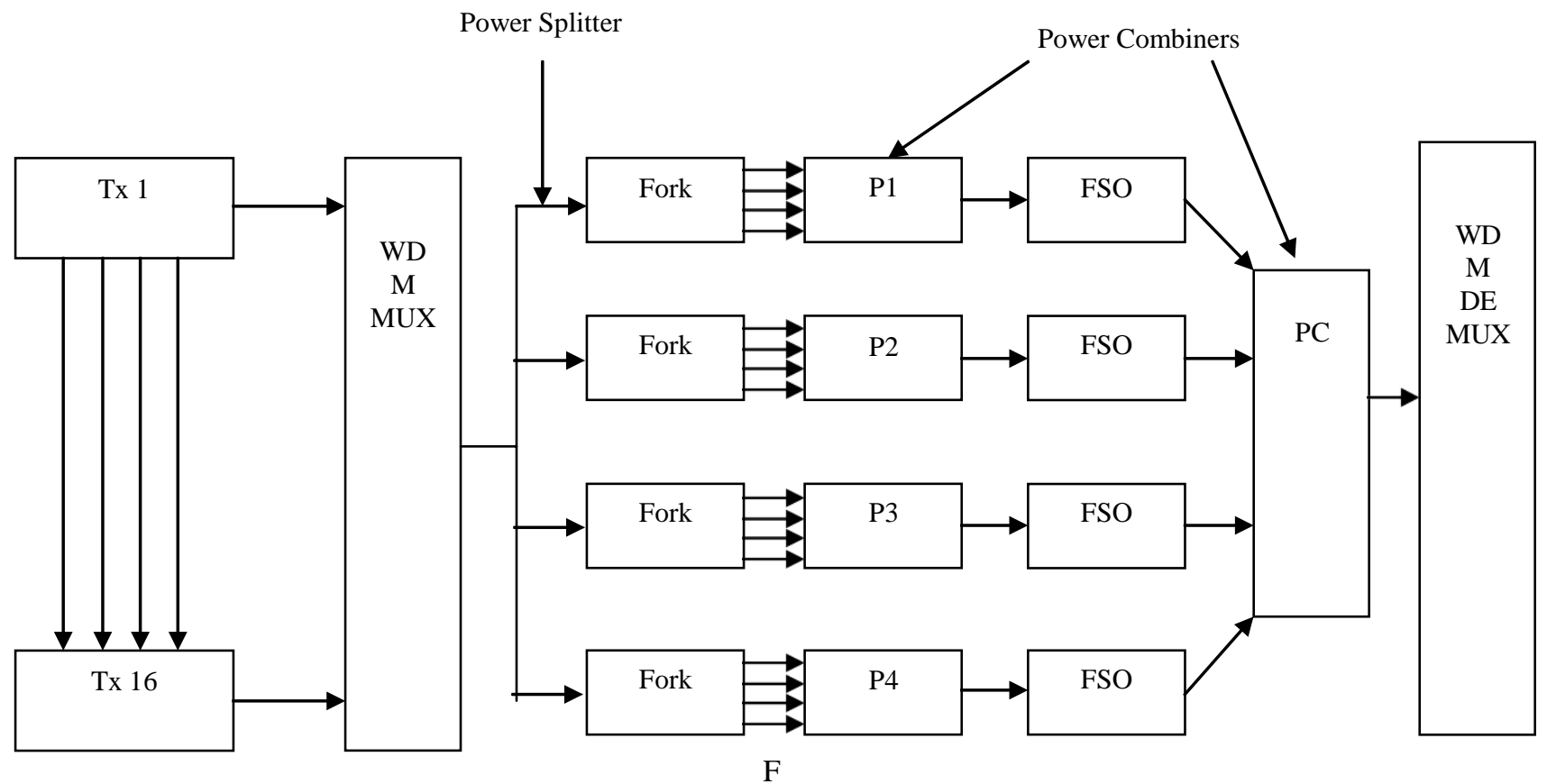

Figure 2: Frame work for the proposed Optical Model

The proposed work is implemented on $32 \mathrm{WDM}$ channel. The motivation behind the proposed work is to get the efficient and capable results which offers better $\mathrm{Q}$ factor and low BER.

\section{Advantages of proposed work over traditional work:} In proposed work the NRZ is replaced with MDRZmodulation and the reason behind this is that the MDRZ is more For the suppression of the signal, modified duobinary return to zero technique has been proposed in the subtractor and delay circuit.

Due to suppression of the signals, there is a proper utilization of bandwidth with no requirement of additional bandwidth supply.

With the adoption of this MDRZ modulation technique, the signals become started producing less noise and other external interference. Therefore the overall result from the system in terms of noise figure and other respective parameters is grown.

\section{RESULTS}

The results achieved after the work on the Optisystem Platform are described in this section. In addition, ananalysis of results of traditional work also analyzes the performance of the work proposed. Usually, the range of BER value lies between 0 and 1 . However, this value should approach to 0 in order to achieve maximum effectiveness of the system. In this section, the simulation results are explained. The graph in figure 3 shows the signal transmitter for traditional operations. advantageous in comparison to NRZ in the following terms:

The $\mathrm{x}$ axis shows the transmitted signal's wavelength and the $\mathrm{y}$-axis displays the signal power.

Figure 3: Transmission Signals in traditional work

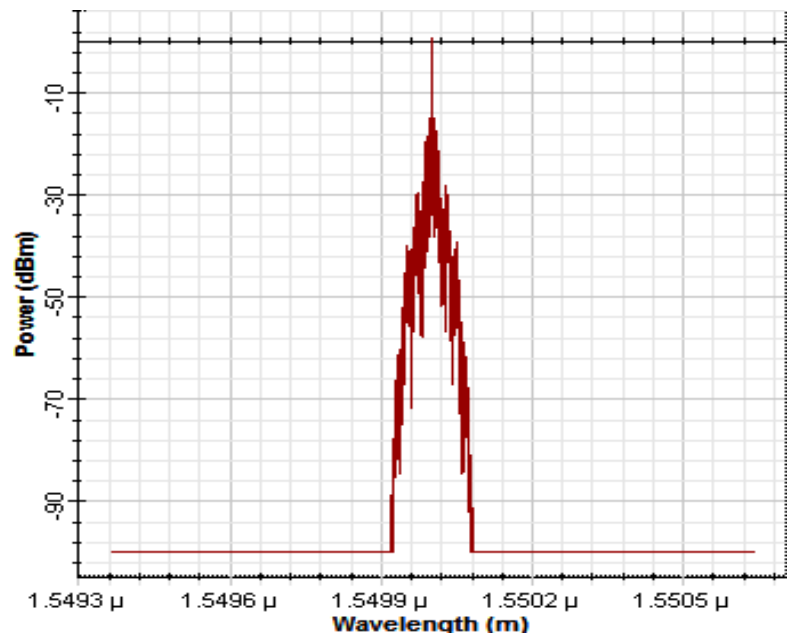

Published By:

DOI: 10.35940/ijitee.I1 105.0789S19

Blue Eyes Intelligence Engineering \& Sciences Publication 


\section{Optical Communication With Wdm Based Fso Over Varying Weather Conditions}

The chart in figure 4 also shows the signal received in the traditional work on the recipient side. $\mathrm{Y}$ axis shows the power of the signal received and ranks between -100 and -40 . The $\mathrm{x}$ axis calibrates the data for the signals as wavelength.

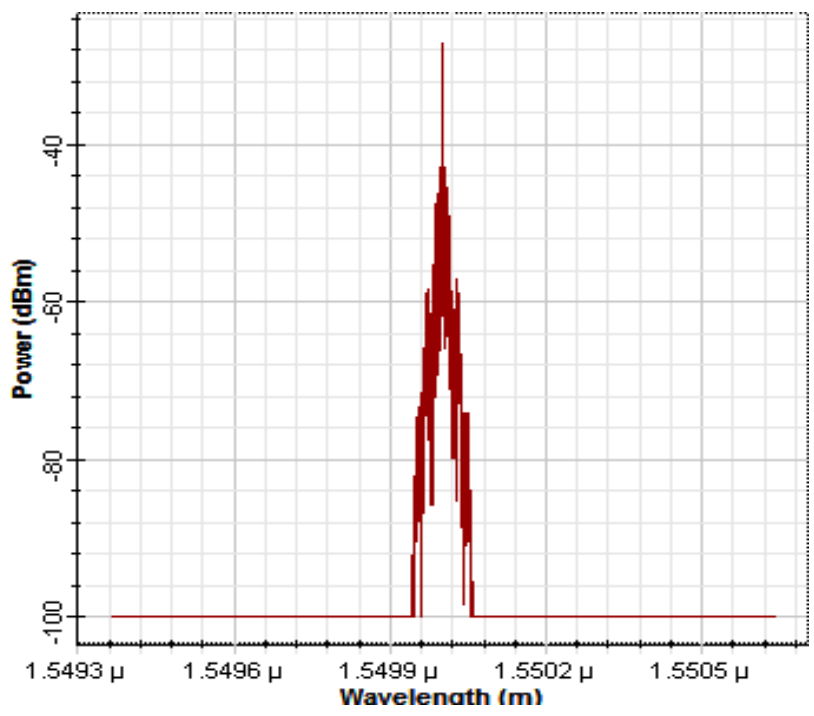

Figure 4: Received Signal in traditional work

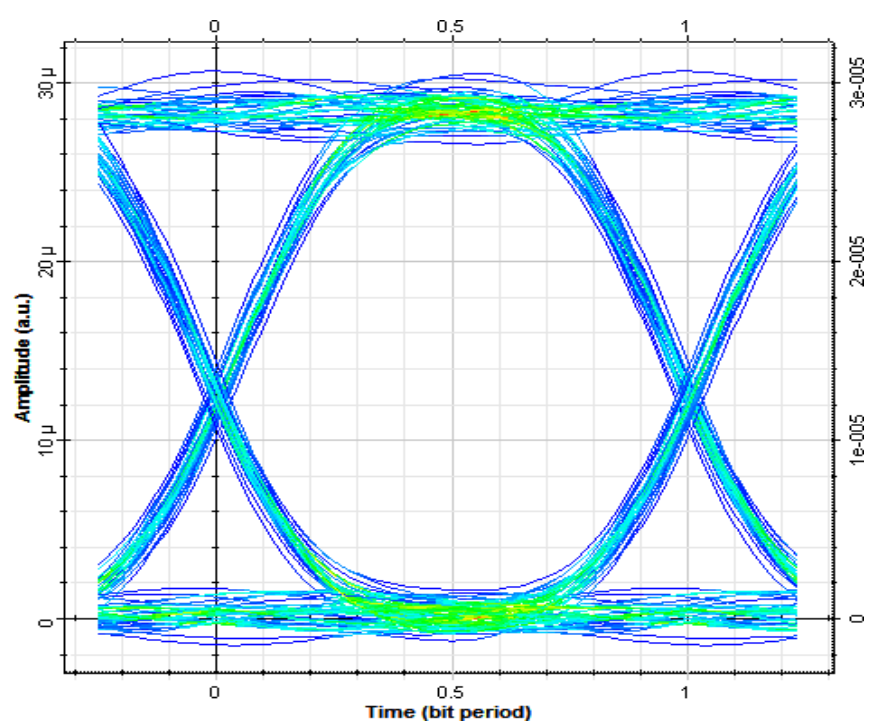

Figure 5: Eye Diagram of traditional work

The eye diagram of the traditional work in Figure 5 shows the graph. It defines many modulated signal multiple. To examine the effect of pulse forming and channel distortion on signals, this is evaluated. Based on time and amplitude, the eye diagram id was created. In figure 6 , the BER for traditional work is described. It should always be a low bit error rate. The BER of traditional work is above 20 according tothe observations in the graph. The maximum Q factor is 21.7985 , min BER is $1.19622 \mathrm{e}-105$, and threshold is $1.32044 \mathrm{e}-005$.

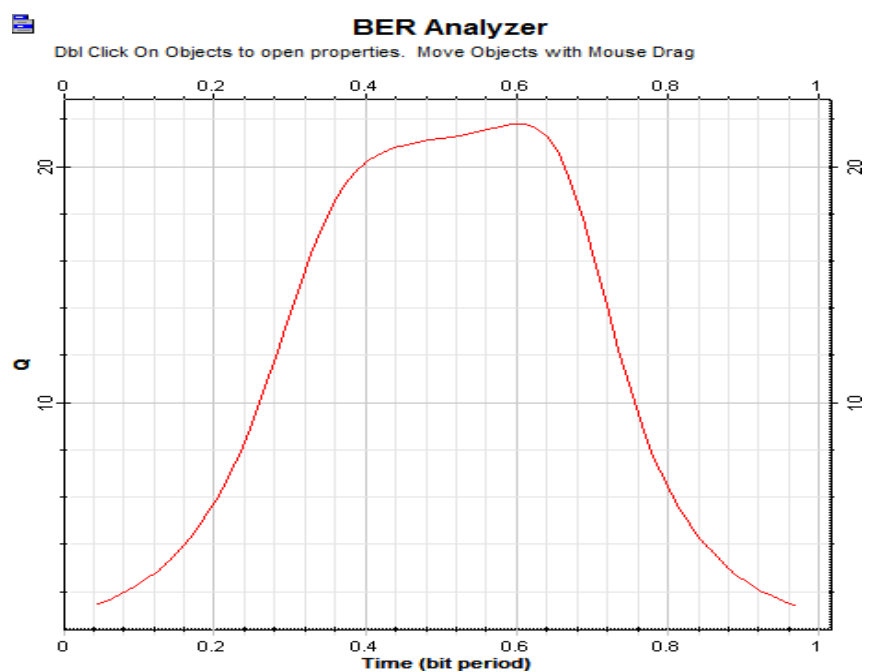

Figure 6: BER of Traditional work

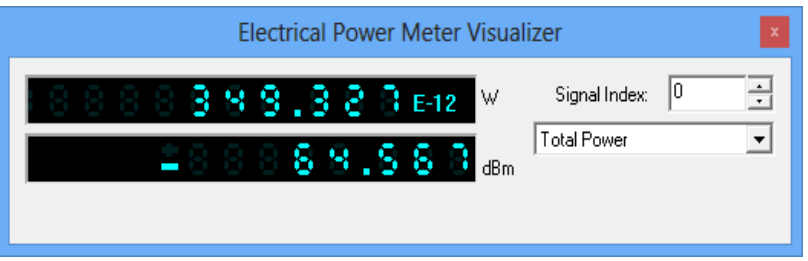

Figure 7: Power of output signal in traditional work

The figure 7 shows the output power of traditional work. The output power of signal in traditional work is $-64.567 \mathrm{dBm}$.

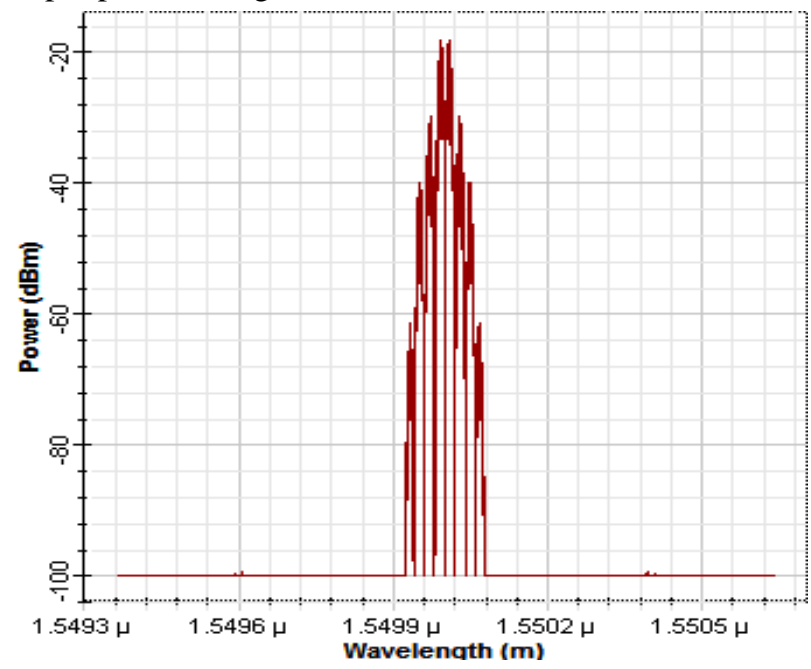

Figure 8 Transmitted signal in proposed work

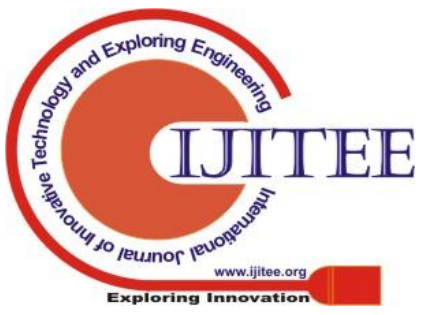




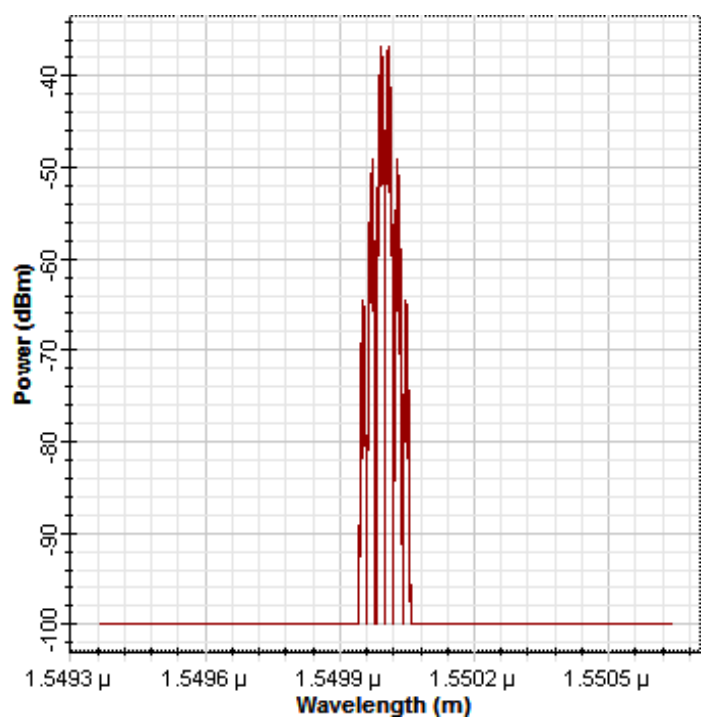

Figure 9: Received signal in proposed work

The graph in figure 8 shows the transmitted signal of proposed work. On the basis of the graph, it is delineated that the power of the transmitted signal in proposed work is $-20 \mathrm{dBm}$. Likewise the graph in figure 9 delineates the graph for received signal in proposed work. The graph presents that the power of the signal that is received in lesser than the power of the signal that is transmitted by thetransmitter.

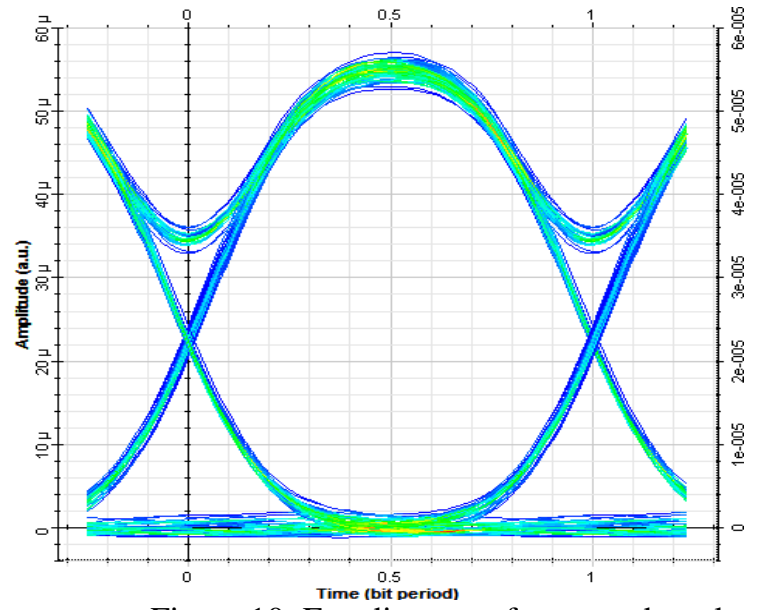

Figure 10: Eye diagram of proposed work

The graph in figure 10 shows the eye diagram of the signal of proposed work. As per the observations from the eye diagram, the max $\mathrm{Q}$ factor of the signal in proposed work is 35.4512, the minimum BER is $1.37259 \mathrm{e}-275$ and threshold is $2.32976 \mathrm{e}-$ 005 .

Simulation results for the proposed model in Optisystem areanalyzed over multiple factors. Quality factor, Bit error rate, Eye height and Total power are performance parameters

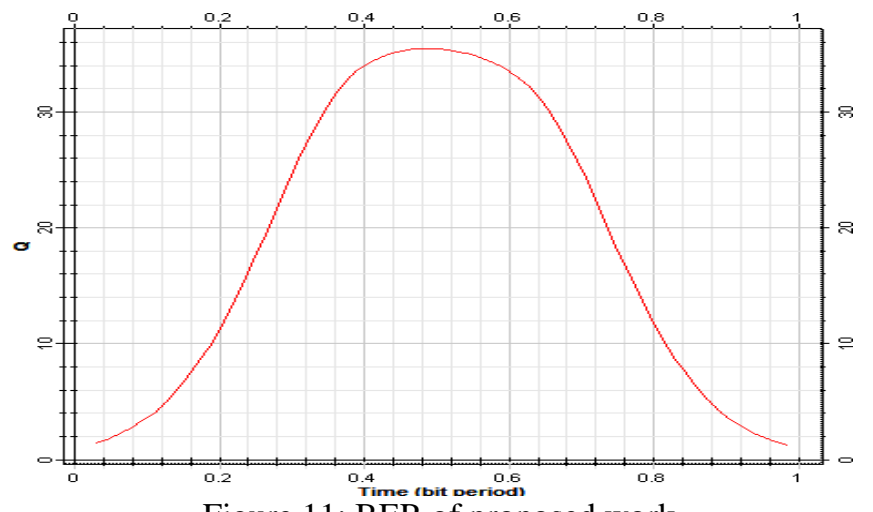

Figure 11: BER of proposed work

The graph in figure 11 represents the BER of proposed work. The BER of proposed work is lower than the BER of traditional work.

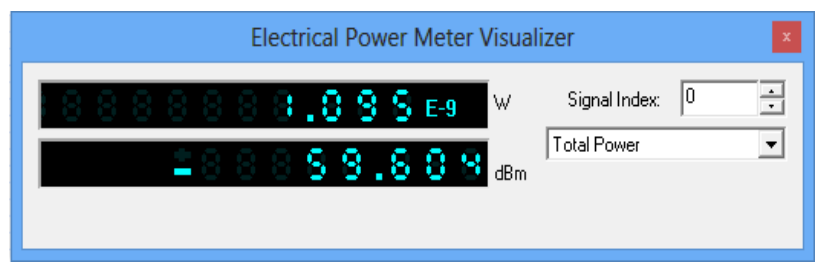

Figure 12: Output power of signal in proposed work

Figure 12 shows the output power of the signal in proposed work. As per the figure, the facts describes that the output power of signal is -59.604 .

After performing the simulation for both traditional and proposed work the results are compared in terms of Quality factor, BER, Eye height and Total power. The comparison results between two models are given below in the Table 1 .

Table 1: Comparison table of traditional and proposed optical model.

\begin{tabular}{|c|c|c|}
\hline Parameters & Traditional & Proposed \\
\hline Quality Factor & 21.7985 & 35.4512 \\
\hline Bit Error Rate & $1.19622 \mathrm{e}-105$ & $1.37259 \mathrm{e}-275$ \\
\hline Eye Height & $2.4324 \mathrm{e}-005$ & $5.00801 \mathrm{e}-005$ \\
\hline $\begin{array}{c}\text { Total Power } \\
(\mathrm{dBm})\end{array}$ & -64.567 & -59.604 \\
\hline
\end{tabular}

\section{CONCLUSION}

those are explaining the upgradation and improvement of the proposed model with respect to existing system. The results shows that the proposed 


\section{Optical Communication With Wdm Based Fso Over Varying Weather Conditions}

model is much effective in terms of Quality factor which is approx 13.65 more than existing system, similarly other factors as the BER, Eye height and Power is improved in the proposed model. As the work done in the proposed model is much effective but still scope of updation will be there, so as a future scope of present work can be done by considering more channels and different data channels.

\section{REFERENCES}

1.Nishu sahu and Jayesh c. Prajapti, "Optimization of WDM-FSO link using Multiple Beams under different rain conditions", Volume 4, Issue 5, May 2015 ,IJARECE.

2.M. Boroon, S. Hitam, M. A. Mahdi, R. K. Z. Sahbudin and S. Seyedzadeh, "Performance of multi-wavelength erbium doped fiber laser on free space optical medium," 2014 IEEE 5th International Conference on Photonics (ICP), Kuala Lumpur, 2014, pp. 99-101. doi:10.1109/ICP.2014.7002323

3. H. Henniger, O. Wilfert, "An Introduction to Free-space Optical Communications", Radio engineering, vol. 19, no. 2, June 2010.

4. X. Cao, "An Integer Linear Programming Approach for Topology Design in OWC Networks," IEEE Globecom Workshops, New Orleans, LA, 2008, pp.1-5.,doi: 10.1109/GLOCOMW.2008.ECP.6

5. Light pointe, "How to design a reliable FSO system", Light pointe white paper series, 2009. Available at: https://www.nebula.wsimg.com

6. S. Bloom, E. Korewaar, J. Schuster, H. Willebrand, "Understanding the performance of free-space optics", Journal of Optical Networking, Optical Society of America, vol. 2, no. 6, June 2003, pp. 178 -200.

7. C. P. Colvero, M. C. R. Cordeiro and J. P. von der Weid, "FSO systems: Rain, drizzle, fog and haze attenuation at different optical windows propagation," SBMO/IEEE MTT-S International Microwave and Optoelectronics Conference, Brazil, 2007, pp. 563-568. doi:10.1109/IMOC.2007.4404328

8.Al Naboulsi, Sizun, and de Fournel," Fog attenuation prediction for optical and infrared waves",Optical Engineering, Vol. 43 No. 2, February 2004,p.319-329.

9. Zvanovec, Stanislav. "Diversity statistics of free space optical links affected by rain" vol 7, pp.407-410.

10. O. Bouchet, H. Sizun, C. Boisrobert, F. De Fornel, P. Favennec, "FreeSpace Optics - Propagation and communication ", ISTE, 2006, pp. $114-$ 119.

11. Mustafa, Fatin \& Supa'at, Abu \& Charde, Nachi Mani, "Effect of Rain attenuations on free space optic transmission in Kuala Lumpur", (Proceeding of the International Conference on Advanced Science, Engineering and Information Technology), 2011, pp. 337 - 341.

12. Fadhil, H.A., Amphawan, A., Shamsuddin, H.A., Abd, T.H., Al-Khafaji, H.M., Aljunid, S.A., \& Ahmed, N.U., "Optimization of free space optics parameters: An optimum solution for bad weather conditions". Optik, 124, 2013pp.3969-3973.

13. Aditi and Preeti, "An effort to design a power efficient, long reach WDMFSO system," 2014 International Conference on Signal Propagation and Computer Technology (ICSPCT 2014), Ajmer,2014,pp.791796.doi:10.1109/ICSPCT.2014.6885013

14.Sharan, Lucky \& G. Shanbhag, Akshay \& Chaubey, Vinod, "Design and simulation of modified duobinary modulated 40 Gbps 32 channel DWDM optical link for improved non-linear performance" Cogent Engineering. 3,2013.

Doi: $10.1080 / 23311916.2016 .1256562$.

15."Fourth-Generation free space optics" available at: https://www.Electronicsforu.com.

16. P. Sathya and S. Robinson, "Impact of Modulation Techniques for HybridWDM Based FSO," 2018 International Conference on Current Trends towards Converging Technologies (ICCTCT), Coimbatore, 2018, pp. 16.

17. Ghazi, Alaan \& Noori, Awab \& Al-dawoodi, Aras \& Aljunid, S \& Syed Idrus, Syed Zulkarnain \& Syed Idrus, Zulkarnain. (2019). Design \& Investigation of $10 \times 10 \mathrm{Gbit} / \mathrm{s}$ MDM over Hybrid FSO link under Different Weather Conditions and Fiber to The Home.

\section{AUTHOR"S PROFILE}

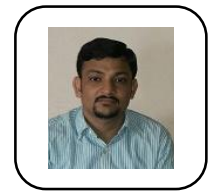

Dr. Rinkesh Mittal is Professor in Electronics and Communication Department .He has $15+$ years of vast teaching experience. He has completed B.Tech, M.Tech. M.B.A. and $\mathrm{PhD}$. His research areas include Wireless Communication,VLSI $\&$ Antenna .He has filed patents \& published $50+$ International Journal papers.

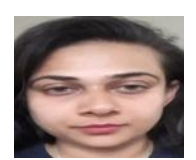

Agrima Kukkarreceived her degree in B.Tech in ECE from DAVIET University in the year 2015. She is currently persuing M.Tech in ECE from Punjab Technical University, Jalandhar. Her research interest are in the field of Wireless \& Optical system

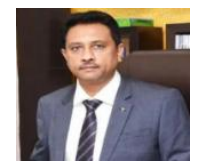

Dr. P.N. Hrisheekesha is a B. E, M. Tech, Ph. D from IIT Roorke. Currently he is working as campus director at CGC Landran. Has got more than 50 publications in reputed international and national journals and conferences. He has completed so many funded projects. He is the editor of few reputed journals. He has guided more than 50 PG and few Ph.Ds. He has few patents and books to his credit.
Published By:

Blue Eyes Intelligence Engineering $\&$ Sciences Publication 